\title{
Clarifying the role of higher-level cortices in resolving perceptual ambiguity using Ultra High Field fMRI
}

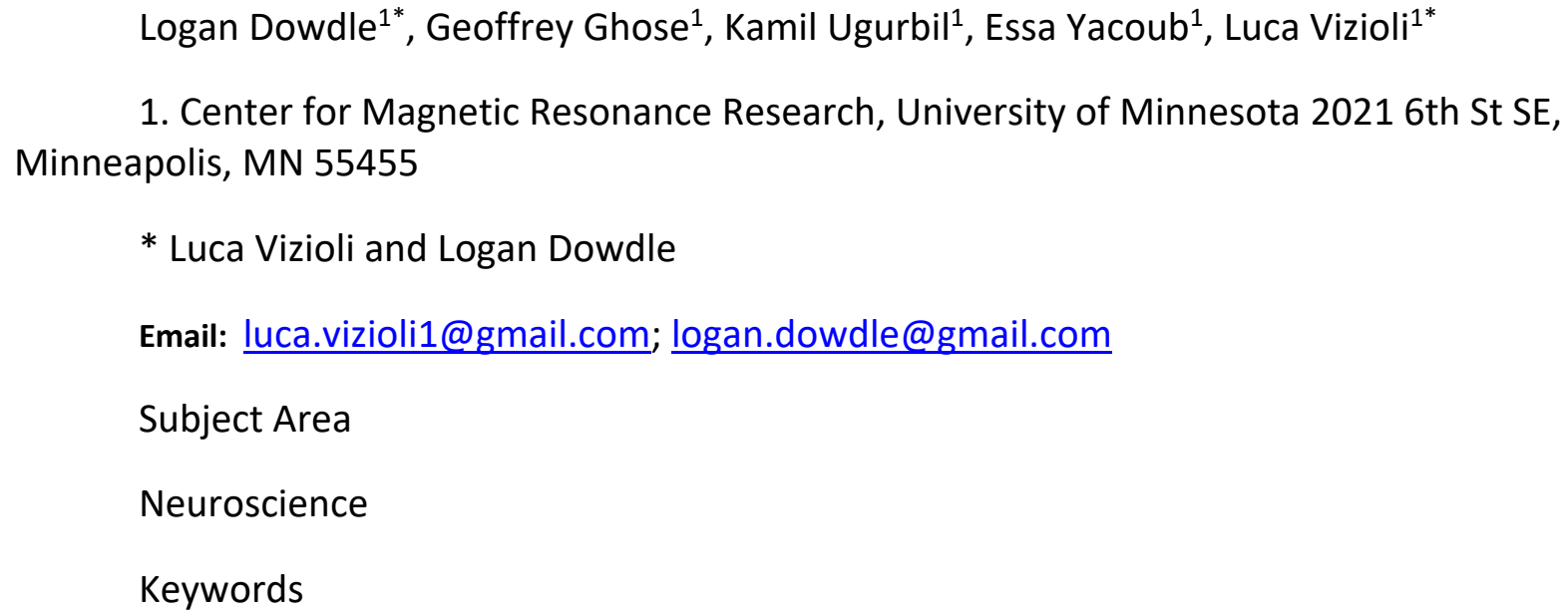

L.V. designed and conducted the experiment; L.V. and L.D. performed the analyses, wrote the manuscript and generated the figures. E.Y. and K.U. provided guidance on choice and optimization of MR acquisition sequences; G.G. provided guidance on conceptual framework; E.Y., K.U. and G.G. contributed to manuscript formulation and writing. 


\section{Abstract}

23 such as attention can modulate sensory representations in accordance with moment-to-moment

24 behavioral requirements. These modulations can be studied by varying task demands; however, the

25 tasks employed are often incongruent with the postulated functions of a sensory system, limiting the

26 characterization of the system in relation to natural behaviors. Here we combine domain-specific task

27 manipulations and ultra-high field fMRI to study the nature of top-down modulations. We exploited

28 faces, a visual category underpinned by a complex cortical network, and instructed participants to

29 perform either a stimulus-relevant/domain-specific or a stimulus-irrelevant task in the scanner. We

30 found that 1. perceptual ambiguity (i.e. difficulty of achieving a stable percept) is encoded in top-down

31 modulations from higher-level cortices; 2 . the right inferior-temporal lobe is active under challenging

32 conditions and uniquely encodes trial-by-trial variability in face perception. 


\section{Introduction}

Decades of human and animal research has demonstrated that cortical areas are functionally and

35 anatomically linked to form distinct brain networks. Understanding the distinct contributions of

36 individual areas within these networks has been challenging in part because of the rich and reciprocal

37 pattern of connections between brain areas (Alexander et al., 1986; Felleman and Van Essen, 1991;

Moeller et al., 2008). Discrimination is further confounded by the low latencies with which signals travel

between areas (Laughlin and Sejnowski, 2003; Wang et al., 2008), substantial overlaps in the functional

sensitivities of neurons of different areas (Arcaro and Livingstone, 2017; Haxby et al., 2001; Vogels and

Orban, 1994), and the flexibility of connections necessary to maintain function across a range of tasks

and environments (Bassett et al., 2011; Gonzalez-Castillo and Bandettini, 2018; Kabbara et al., 2017). A

critical component of this flexibility in sensory systems is top-down control, in which cognitive variables

such as attention, value, and memory can alter sensory representations in accordance with explicit or

specific behavioral demands. Yet this flexibility also is a major challenge to experimental studies, because

it implies that the nature of signal processing both within and between brain areas of a network is not

47 fixed and very much depends on the behavioral context and goals of the subject.

While many humans and animal studies have demonstrated the ability of task manipulations to

modulate sensory evoked responses, in most cases these studies have employed tasks that are not

50 specific to the domain of the stimulus that was presented. For example, numerous electrophysiological

51 and imaging studies suggest that attending to a particular location in visual space increases the

52 responses of neurons whose receptive fields lie within the attended location irrespective of the

53 selectivities of those receptive fields (Cohen and Maunsell, 2011; Liu et al., 2015; Martinez-Trujillo and

54 Treue, 2004). Similarly, perceptual tasks involving working memory often result in a broad distribution

55 of enhanced signals in sensory areas (Druzgal and D’Esposito, 2003, 2001; Kay and Yeatman, 2017; Leung 
56 and Alain, 2011; Pessoa et al., 2002). By contrast, top-down modulation from domain-specific tasks can

57 be far more targeted, selectively enhancing only those neurons whose receptive field responses are

58 consistent with behavioral demands (De Martino et al., 2015; Zhang and Kay, 2020). Accordingly, the use

59 of appropriate domain specific tasks is likely to highlight those neurons with relevant selectivities and

60 reveal how they contribute to perception. For example, contrasting the activity elicited by identical face

61 stimuli in an $\mathrm{N}$-back and a fixation task may reveal working memory related modulations that are

62 unspecific to face processing and therefore fail to elucidate the changes related to the specialized

63 processing of faces. Indeed, a N-back face task could be performed without explicit face recognition or

64 perception; additionally, one could imagine that explicit vs. implicit face detection may be underpinned

65 by different neural computations, and therefore, implementing a task with no explicit requirement may

66 represent a confound or lead to a different set of results. entire network and then identifying the particular areas that can best explain task performance.

71 techniques are frequently performed in non-human primates, with tasks that fail to capture the cognitive

72 sophistication available to humans. Moreover, even whole brain studies, such as those relying upon

73 functional magnetic resonance imaging, are often highly biased in their sampling because of the difficulty

74 of imaging particular areas. For example, a number of high-level areas - crucial for human cognition -

75 are located in cortical loci that are traditionally difficult to image (e.g. due to low SNR and their proximity

76 to air cavities (Chen et al., 2003; Devlin et al., 2000)). These limitations also constrain our ability to

77 establish appropriate animal models for invasive measurements and manipulations because of the

78 possibility that human areas homologous to those in animal studies are difficult to sample with

79 conventional gradient echo fMRI methods (Rajimehr et al., 2009). Signal to noise issues have also limited 
80

81

82

83

84

85

86

87 the ability of $\mathrm{fMRI}$ to establish trial-to-trial behavioral covariance, which is a critical requirement for understanding the neural basis of perception (Parker and Newsome, 1998) and has been extensively employed by electrophysiology studies (Desimone et al., 1984; Hubel and Wiesel, 1959; Martinez-Trujillo and Treue, 2004; Perrett et al., 1982). This is a particularly important consideration because, by definition, all areas within a network are co-activated, but, given the richness of connectivity within the network, that co-activation does not necessarily imply perceptual relevance.

While the difficulties surrounding magnetic field inhomogeneity and transmit uniformity can be more detrimental at higher fields, there are substantial SNR gains available with ultra-high field magnets (UHF i.e. 7T and above). In common practice, these SNR gains are generally traded for higher resolutions (e.g. submillimeter). In this paper we instead select a relatively lower resolution (i.e. $1.6 \mathrm{~mm}^{3}$ ) and leverage the improved SNR available in UHF fMRI to study the entire ensemble of brain areas associated with a behaviorally important high-level perceptual task, namely the detection of faces in poor visibility. We choose faces as these are a well-studied, cross species visual category that is highly meaningful and therefore ideal for investigating high-level, top-down neural modulations. The human face processing network has however proved challenging to study (Kanwisher et al., 1997; Kanwisher and Yovel, 2006; McCarthy et al., 1997) (see Grill-Spector \& Weiner 2014 for review), because many of its areas, due to their proximity to air cavities, are heavily affected by the aforementioned fMRI signal difficulties. In order to combat susceptibility related dropout we leverage the improved acceleration available at high fields to increase our imaging resolution to $1.6 \mathrm{~mm}$ relative to the $2 \mathrm{~mm}$ gold-standard for $3 \mathrm{~T}$ studies. Combined with manual shimming, this yields substantial gains in SNR, while reducing drop out (Farzaneh et al., 1990; Olman et al., 2009; Young et al., 1988) in key human face processing areas. In the current work, we varied the visibility of face stimuli by modulating their phase coherence (Figure 1) and instructed the subjects to perform 2 tasks in the scanner: a domain-specific, stimulus relevant task involving perceptual judgment of the visual stimuli (i.e. face detection); and, stimulus irrelevant fixation task. By leveraging 
bioRxiv preprint doi: https://doi.org/10.1101/2020.05.27.119677; this version posted November 17,2020 . The copyright holder for this preprint (which was not certified by peer review) is the author/funder, who has granted bioRxiv a license to display the preprint in perpetuity. It is made available under aCC-BY-NC-ND 4.0 International license.

104 signal gains derived from our imaging approach, we then contrast these responses to isolate top-down

105 modulations specific to the face domain and link subjective perception to brain activity.

106 


\section{Materials and Methods}

Psychophysics Toolbox (3.0.15) based code. Participants viewed the images though a mirror placed in

121 the head coil. Behavioral responses were recorded using Cambridge Research Systems button box and 122 Psychophysics Toolbox.

\section{Participants}

10 (5 females) healthy right-handed subjects (age range: 18-31) participated in the study. Of these, 1 participant was re-scanned due to excessive motion during scanning. All subjects had normal, or corrected vision and provided written informed consent. The local IRB at the University of Minnesota approved the experiments.

\section{Stimuli and procedure}

The experimental procedure consisted of a standard block design face localizer and a fast eventrelated face paradigm. For both experiments, the stimuli were centered on background of average luminance $(25.4 \mathrm{~cd} / \mathrm{m} 2,23.5-30.1)$. Stimuli were presented on a Cambridge Research Systems BOLDscreen 32 LCD monitor positioned at the head of the 7T scanner bed (resolution 1920, 1080 at 120 $\mathrm{Hz}$; viewing distance $\sim 89.5 \mathrm{~cm}$ ) using a Mac Pro computer. Stimulus presentation was controlled using

All visual stimuli used for the face localizer consisted of grayscale photographs depicting 20 127 different faces (10 identities $\times 2$ genders) and objects (both taken from Stigliani et al., 2015) and textures 128 of noise. 

categories. There were 11 categories, grouped into 3 stimulus domains: faces (adult, child), no face

(Willenbockel et al., 2010). The root mean square contrast (i.e. the standard deviation of pixel intensity)

was also kept constant across stimuli.

\section{Visual presentation paradigm}

Face localizer runs involved presentation of blocks of faces, objects and noise textures. Each run

144 began with presentation of a black fixation cross displayed on a grey background for 12 sec and consisted

145 of 9 randomly presented blocks of images. Each block ( 3 blocks/category; separated by a 12 sec fixation)

146 involved presentation of 10 different stimuli randomly presented for $800 \mathrm{~ms}$, separated by a $400 \mathrm{~ms}$

147 interstimulus interval (ISI). To ensure that participants' attention was maintained throughout the

148 localizer we implemented a 1-back task, where subjects were instructed to respond to the repetition of

1492 identical stimuli (representing about $10 \%$ of the trials), by pressing a button on a response pad held in

150 their right hand. All participants completed two runs of the block design face localizer, where each block

151 occurred 3 times within a run, for a total run duration of 228 seconds. 


\section{Event related experiment.}

\section{Stimuli}

We used grayscale images of faces (20 male and 20 female), presenting neutral expressions. We

157 (5 visual conditions $\times 20$ identities $\times 2$ genders). We equated the amplitude spectrum across all images.

158 Stimuli approximately subtended $9^{\circ}$ of visual angle. Faces were cropped to remove external features by

159 centering an elliptical window with uniform gray background to the original images. The y diameter of

160 the ellipse spanned the full vertical extent of the face stimuli and the $x$ diameter spanned $80 \%$ of the

161 horizontal extent. Before applying the elliptical window to all face images, we smoothed the edge of the

162 ellipse by convolving with an average filter (constructed using the "fspecial" function with "average"

163 option in MATLAB - see Figure 1A). This procedure was implemented to prevent participants from

164 performing edge detection, rather than the face detection task, by reacting to the easily identifiable 165 presence of hard edges in the face images.

167 stimulus set. We controlled the Fourier spectra across stimuli, ensuring that the rotational average

168 amplitudes for a given spatial frequency were equated across images while preserving the amplitude

169 distribution across orientations (Willenbockel et al., 2010). The root mean square contrast (i.e. the 170 standard deviation of pixel intensity) was also kept constant across stimuli. 
In the scanner, participants were instructed to maintain fixation on a central cross throughout

174 the run and to perform one of 2 tasks: one domain-specific face detection task, involving perceptual

175 judgment of the visual stimuli; and a second, difficult, non-specific attention fixation task that required

176 responding to a specific color change (i.e. red) of the fixation cross.

In the former, participants were instructed to respond as quickly as possible by pressing one of 2

buttons on their button box to indicate whether they perceived a face. Subjects' instructions were

carefully delivered to indicate that there were no correct answers and that we were instead interested

in the subjects' perception only (Figure 1B).

The latter was designed to isolate bottom-up stimulus-driven responses. In order to ensure this,

we piloted the fixation task to maximize task difficulty and direct the attention away from the face stimuli

on 4 participants that were not included in the experimental subject pool. Based on participants'

184 feedback, the fixation tasks entailed pressing a button every time the fixation turned red. Every 500 ms,

the fixation changed to one of five colors - specifically red, blue, green, yellow and cyan - in a

pseudorandom fashion, avoiding consecutive presentations of the same color (Figure 1C). The color

change occurred out of sync with the stimulus presentation onset and frequency of button presses was

kept constant across tasks. Visual stimuli were identical across tasks to ensure that any differences

observed were related solely to top-down processes. Tasks were blocked by run and counterbalanced across participants.

Visual presentation paradigm.

We acquired 3 runs per task. Each run lasted approximately 3 mins and 22 secs and began and 
196 Importantly, we introduced $10 \%$ blank trials (i.e. $4000 \mathrm{~ms}$ of fixation period) randomly interspersed

197 amongst the 40 images, effectively jittering the ISI. Stimulus presentation was pseudorandomized across

198 runs, with the only constraint being the non-occurrence of 2 consecutive presentations of the same

199 phase coherence level. Behavioral metrics, including reaction time and responses to face stimuli

200 indicating participants' perceptual judgments (i.e. face or no face) were generated per subject and then

201 averaged.

202

203

\section{Ambiguity Calculation}

204

For the face detection task, we calculated the average face response percentage to each

205

phase condition, averaged across runs. These values vary from 0 to $100 \%$, representing consistent non-

206 face to consistent face response respectively. Within each subject, this produces a sigmoidal shaped

207 curve. We mathematically define the ambiguity score, on a subject by subject basis, as the inverse of the

208 absolute distance from the inflection point on the sigmoid that fit this curve This is shown in Equation 1:

209

Eq 1. $\left(\left|S_{i}-S_{k}\right| \times-1\right)+\min (A)$

where $S_{i}$ is the $i$ th point of the faceness sigmoid, $S_{k}$ is the theoretical midpoint of the faceness

211 sigmoid and $\min (\mathrm{A})$ is the minimum value of the ambiguity function. While conceptually similar to

212 defining a perceptual threshold, the goal of this analytical approach is to characterize the instability of

213 the percept in a threshold-free, data-driven manner for all visual conditions, rather than determining the

214 precise level of phase coherence information required to achieve a reliable face percept.

\section{MR Imaging Acquisition and Processing}

All functional MRI data were collected with a 7T Siemens Magnetom System with a 1 by 32-

217 channel NOVA head coil. T2*-weighted images were collected using sequence parameters (TR 1s, 
218 Multiband 5, GRAPPA 2, 7/8ths Partial Fourier, 1.6mm isotropic, TE 22ms, Flip Angle 52 ${ }^{\circ}$, Bandwidth

$2191923 \mathrm{~Hz}, 85$ slices, FOV $208 \times 208 \mathrm{~mm}$ ) matched to the Human Connectome 7T protocol (Thanh Vu et al., 220 2017). shimmed the BO field to maximize homogeneity over ventral and anterior-temporal regions. Moreover, we selected our flip angle based on the offset between the selected flip angle and the subject specific

224 flip angle, using the results from a 3dAFI (Actual Flip Angle Image) sequence. In this way we optimized 225 flip angles across the brain to maximize SNR in the ventral and anterior-temporal regions. Finally, by 226 taking advantage of the greater ability to accelerate image acquisition at 7T, we were able to obtain 227 higher resolution images and relative reductions in magnetic susceptibility dropout in these regions 228 (Farzaneh et al., 1990; Olman et al., 2009; Young et al., 1988). ms; FOV, $256 \times 256 \mathrm{~mm}$; flip angle, $9^{\circ}$; TE, $2.52 \mathrm{~ms}$; spatial resolution, .8 mm isotropic voxels) which were collected with a 3T Siemens Magnetom Prisma ${ }^{\text {fit }}$ system. Anatomical images were used for visualization purposes only.

\section{Functional Image Processing} processing was performed in AFNI version 19.2.10 (Cox, 1996). Conventional processing steps were used, including despiking, slice timing correction, distortion and motion correction, and alignment to each participant's anatomical image. With each task type, the target for time series and anatomical alignment was the Single Band Reference (SBRef) image which is acquired to calibrate coil sensitively profiles prior 
241 et al., 2013). In order to improve localizer and task alignment, an additional nonlinear transform was

242 computed between the task SBRef and localizer SBRef. All spatial transforms were concatenated and

243 applied in a single step to reduce image blurring, and functional images were inspected to confirm

244 successful registration to anatomical targets.

For the functional localizer, the data was then smoothed with a Gaussian kernel with FWHM of

\section{Functional ROI Definition}

Using 3dDeconvolve, the data were passed through a GLM in order to determine the response

254 to faces, objects and noise. Each event was modeled as a 12s box car, convolved with AFNI's SPMG1 HRF

255 estimation. Rather than select face patches by comparing face activation to the average of objects and

256 noise, we instead constrained the statistical map in the following ways: 1) Betas to faces were positive,

257 2) the T-stat used for thresholding was the minimum positive T-stat from faces $>$ objects or faces $>$ noise.

258 In other words, we selected voxels that were significant for faces greater than objects, in conjunction

259 with faces greater than scrambled. Regions of interest (ROIs) were derived in volume space, and all

260 consisted of contiguous clusters made up of 19 or more voxels. Statistical thresholding was adjusted to

261 obtain consistency between subjects, no voxels above $p<0.05$ were considered. To aid in ROI definition,

262 we simultaneously viewed the surface representation of the statistical map using SUMA (Saad and 
263 Reynolds, 2012) with FreeSurfer (Dale et al., 1999; Fischl et al., 2004, 2002, 2001) defined cortical

264 surfaces and atlas labels. In addition, to supplement the statistical parametric maps, we used finite

265 impulse response (FIR) deconvolution, with 20 bins, to estimate HRFs in response to each localizer

266 condition (face, scrambled, object). These HRFs were also used to inspect data quality for each ROI.

For the Face/Fix detection tasks, the data were only scaled following initial processing, no

270 smoothing was used. As we were interested in BOLD responses across the whole brain, and we know

271 that hemodynamic response functions differ across cortical regions (Handwerker et al., 2004; Lewis et

272 al., 2018; Taylor et al., 2018), we performed a GLM based finite impulse response (FIR) deconvolution

273 analyses, which does not make assumptions regarding the shape of the HRF. This was done using AFNI's

274 TENTZero method, estimating responses with $1 \mathrm{~s}$ bins out to $15 \mathrm{~s}$ post stimulus. Deconvolution was

275 performed separately for each task (face detection and fixation) and phase coherence condition,

276 generating 5 FIR curves for the fixation task and 5 for the face detection task. For each task and

277 independently per subject, ROI, run and condition, we averaged all FIR curves across all voxels. To avoid

278 the contribution of extreme voxels, we trimmed $5 \%$ of the values falling at each extreme of the

279 distribution tail to compute the $10 \%$ trimmed mean. We then extracted the 4 timepoints corresponding

280 to 4, 5, 6 and 7 seconds after stimulus onset, i.e. those with the largest amplitude within a time window

281 spanning from 2 to 10 TRs after stimulus onset. The 12 extracted amplitudes (4 timepoints per run) were

282 then averaged within experimental tasks to obtain one percent signal change value per subject, ROI, task

283 and condition. 
To test for statistically significant differences between conditions (i.e. phase coherence levels) and tasks, we carried out the following statistical tests.

\section{Task and Condition Analyses}

Independently per ROI, we performed a 2 (tasks) by 5 (phase coherence levels) repeated

centering the data on the group mean for each condition and task. This procedure effectively put the

data under the ideal $\mathrm{HO}$ hypothesis of no difference between the means. We then sampled with

replacement the subjects and performed the same $2 \times 3$ repeated measures ANOVA and stored all $F$

values for the relevant main effects and interactions. We repeated this procedure 10,000 times and

selected the $95 \%$ largest $F$ values. We used these $F$ values as our new thresholds and considered

statistical significance only when $\mathrm{p}$ values for the original ANOVA were smaller than .05 and the

connected F values were larger than their centered counterpart (e.g. (Wilcox, 2005))

When appropriate (i.e. for comparisons entailing more than 2 factors), we further performed

post-hoc paired sample t-tests on significant (as defined above) main effects and interactions. The same

FWER control procedure described above for the ANOVA test was implemented to account for multiple comparisons.

Additionally, we performed power analyses to determine effect size and the sample size required

to achieve adequate power (see results). Specifically, we computed Hedges' g (Freeman et al., 1986;

306 Hedges, 1981). This choice was motivated by the fact that, unlike Cohen's d, which, especially for small

307 samples (i.e. $n<20$ ), tends to provide positively biased estimators of population effect sizes (Freeman

et al., 1986; Lakens, 2013), Hedges' g tends to be unbiased (Cumming, 2012). 
To determine the extent to which task demands modulate functional connectivity amongst face

312 ROIs, we carried out the following analysis. Independently per subject, task and ROI, we averaged (mean)

313 all FIR response curves amongst voxels and concatenated the time courses elicited by each condition to

314 produce a single time course. The concatenation was done to maximize statistical power, as the resulting

315 time course contained 80 timepoints (i.e. 16 FIR timepoints $\times 5$ conditions). For each subject and task,

316 we then computed Pearson's correlation coefficient between the concatenated time courses of all

317 possible pairs of ROls. This procedure lead to the formulation of 2 connectivity matrices (12 ROIs x 12

318 ROIs) per subject (1 per task) summarizing the similarity of BOLD responses between all pairs of ROIs. To

319 convert the skewed sampling distribution of Pearson's $r$ into a normal distribution we computed Fisher's

$320 \mathrm{z}$ transformation (Fisher, 1915). We therefore proceeded to carry out paired sample t-tests between the

321 connectivity estimated obtained for the 2 tasks. To control for FWER, we implemented the same

322 bootstrap procedure on centered data described in the previous paragraphs (see paragraph 4.6.1). For

323 display purposes only, after computing the mean between the Fisher z-normalized connectivity matrices,

324 we computed the inverse of such transformation on the group average connectivity matrices to convert

325 these scores back into meaningful and interpretable Pearson's r.

To better visualize the results of our functional connectivity analysis, we further performed

327 classic multidimensional scaling (MDS - using the function "cmdscale" in MATLAB) on the participants

328 average dissimilarity matrix (i.e. 1- Pearson's r). MDS was performed independently per task. For ease

329 of visual comparison, the MDS arrangements of the 2 tasks were aligned by means of linear

330 transformations (including translation, reflection, orthogonal rotation, and scaling) using Procrustes 
331 rotations (this was implemented with the "procrustes" function in MATLAB) with the sum of squared

332 errors as stress metric.

MDS is a useful data driven approach to visualize the data projected into a new space whose

334 dimensions are the first (in this case 2) eigenvectors (i.e. those explaining most of the variance in the

335 data) without any prior hypotheses. MDS is therefore a dimension reduction technique that highlights

336 the dominant features in the data. Results are shown in Figure 4; distance between the points indicate

337 dissimilarity of responses.

Brain-behavior correlation.

Next for each ROI, we wanted to assess the relationship between behavioral responses and top-

341 down BOLD modulations. To this end, for each subject and ROI, we computed Pearson's correlation

342 coefficients amongst the ambiguity scores at each phase coherence level and the task difference in

343 average BOLD amplitudes elicited by each condition. We then performed Fisher z transform (see

344 paragraph above) on Pearson's $r$ and carried out one sample t-test for each ROI to determine whether

345 the average group correlation was significantly larger than 0 . To control the family wise error rate

346 (FWER), we implemented the same bootstrap procedure on centered data described in the previous

347 paragraphs (see paragraph 4.6.1). For display purposes only, after computing the mean between the

348 Fisher z-normalized correlation scores, we computed the inverse of such transformation on the group

349 average to convert these scores back into meaningful and interpretable Pearson's r. 
bioRxiv preprint doi: https://doi.org/10.1101/2020.05.27.119677; this version posted November 17,2020 . The copyright holder for this preprint

(which was not certified by peer review) is the author/funder, who has granted bioRxiv a license to display the preprint in perpetuity. It is made available under aCC-BY-NC-ND 4.0 International license.

We performed additional analyses to assess BOLD amplitude modulations as a function of

353 percept. Due to the grouping of responses, we considered only the most ambiguous condition (i.e. 20\%

354 phase coherence, see results). We allocated each trial to one of 2 new conditions: "face percept" and

355 "no face percept" on the basis of each participants reported percept. We then repeated the analysis

356 described in section 2.5.4 to estimate BOLD amplitudes of these 2 conditions. 


\section{Results}

360 hemispheric lateralization.

Localizer Task.

Using the separate face-localizer task, we identified a total of twelve ROIs for all participants. This

364 included typical regions, such as the fusiform face area (FFA, MNI Centers of Mass [L: -41 -52 -19, R: 40 -

$36554-17]$ ), occipital face area (OFA [L: -44 -83 -13, R:43 -76 -11]), posterior superior temporal sulcus (STS

366 [L: -48 -68 10, R: $50-5718]$ ) as well as the amygdalae [L: -21 -7 -15, R: $20-6-15]$. In addition, we identified

367 ROIs for more difficult regions, including an ROI proximal to the perirhinal cortex, which we are referring

368 to more generally as the anterior inferior temporal (AIT [L: $-34-9-34, \mathrm{R}: 32-6-39]$ ) cortex as well as an

$369 \mathrm{ROI}$ in H-shaped sulcus, which we refer to as the HFA (H-shaped sulcus Face Area, [L: $-3233-15, \mathrm{R}: 3033$

$370-14])$. ROls identification and all subsequent analyses were carried out in native subject space, but to

371 compare their locations across participants and to relate these locations to previous studies, we

372 converted and report their coordinates in MNI space. All cortical regions and their overlap (in the specific

373 slices selected) across participants are shown in Figure 2A. 

available under aCC-BY-NC-ND 4.0 International license.

A.

Experimental Stimuli

B.

Stimulus Relevant

Face Detection Task

C.

Stimulus Irrelevant Fixation Task

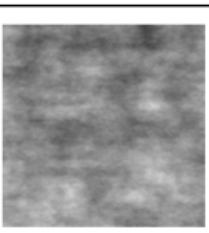

$0 \%$

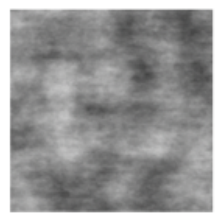

$10 \%$

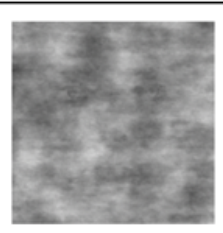

$20 \%$

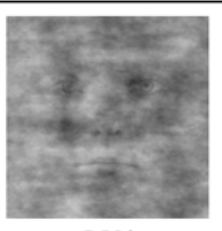

$30 \%$

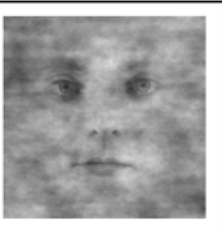

$40 \%$

Phase Coherence

2s grey, with fixation color changes at $2 \mathrm{~Hz}$

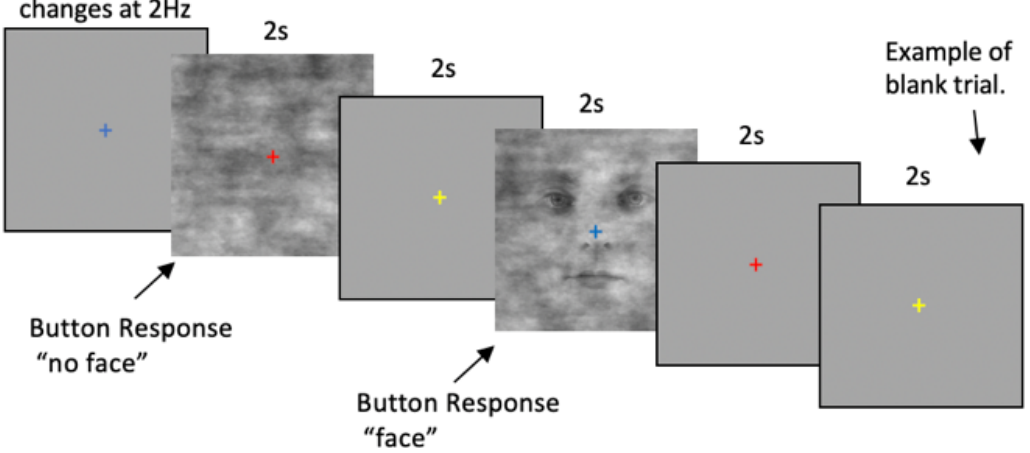

2s grey, with fixation color changes at $2 \mathrm{~Hz}$
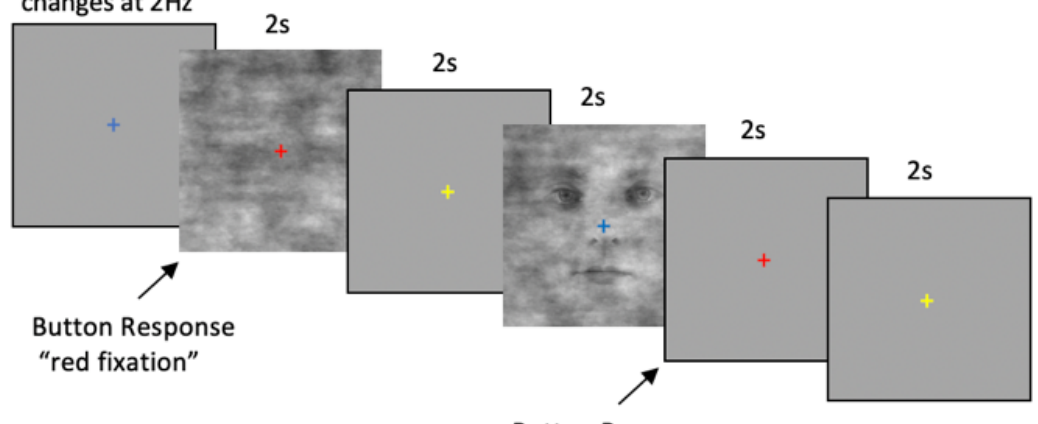

Button Response "red fixation"

Figure 1. Event Related Stimuli and Tasks. A) Example stimuli associated with the 5 phase coherence levels used. B) The Stimulus Relevant Face detection task. Stimuli appeared for 2 seconds, with an inter-trial interval of 2 seconds. The fixation cross changed color with a frequency of $2 \mathrm{~Hz}$ and was visible throughout all experimental procedures. Participants indicated "face" or "no face" using an MRI response hand pad. Blank trials were randomly inserted during each fMRI run. C) The Stimulus Irrelevant Fixation task. Timing is identical to B. Participants indicated when the fixation cross changed to the color red. 


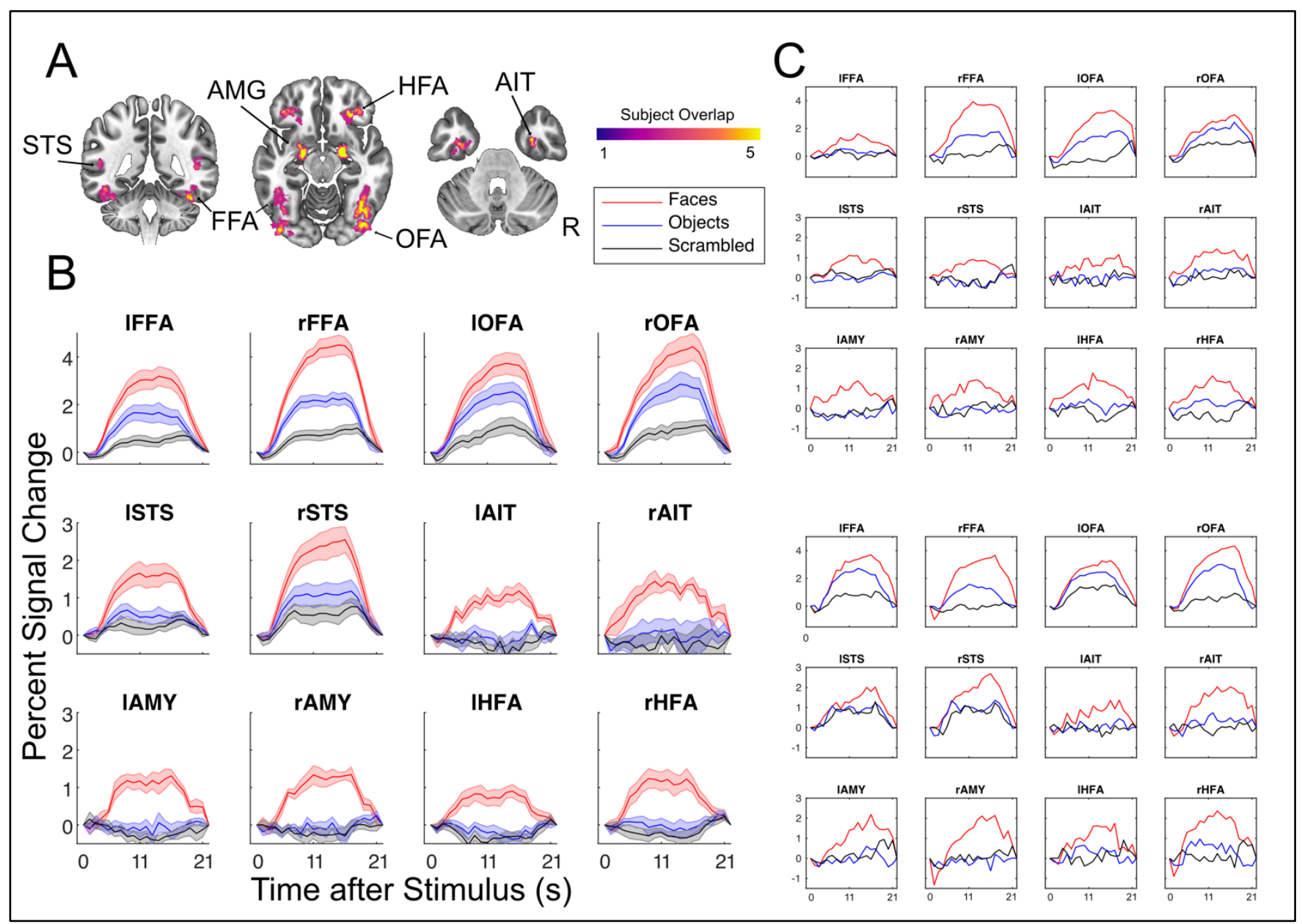
data quality.

Figure 2. Results from the face preferential localizer. A) All subject's ROIs combined in MNI template space, highlighting the consistent overlap between subjects and between hemispheres. Labels indicate ROI names, right is right. B) Average BOLD responses across all subjects, in units of percent signal change, for each ROI in response to faces, objects and scrambled images. Standard Error of the Mean shown with shading. C) Example results from two single subjects showcasing reliability even within a single subject's ROls.

Figure 2B shows ROls average BOLD time courses, constructed from FIR models. All regions, including areas associated with low SNR (AIT, HFA), yield plausible hemodynamic responses. Furthermore, these responses remain HRF-like even at the single subject level (Figure $2 \mathrm{C}$ ), indicating high 

responded with an average RT of 760 +/- 120 ms. Reaction times indicate that, in reporting their percepts, participants were slowest at the lower end of the phase coherence spectrum (i.e. $0 \%$ and $10 \%$ ),

401 becoming increasingly faster as a function of phase coherence. phase coherence (Figure 3A, right panel). 


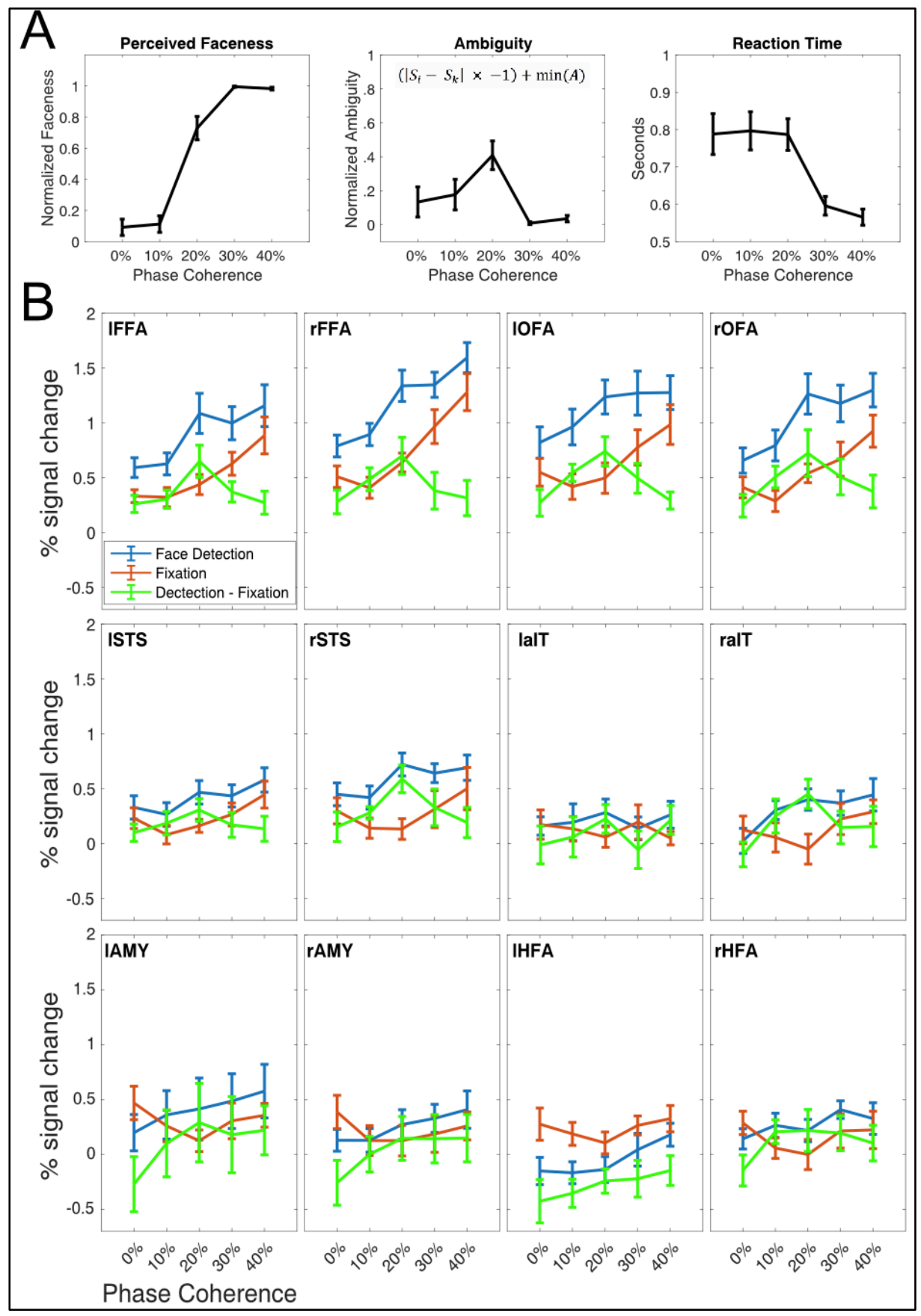

Figure 3. A) Behavioral Responses to Stimulus Relevant Task. Left panel: The group average of perceived "faceness", where 0 represents consistently reporting no face, and 1 represents consistently reporting the presence of a face. Central Panel: The group averaged ambiguity curve, showing that stimuli at $20 \%$ were most ambiguous, i.e., were most inconsistently categorized. Ambiguity was computed according to the equation shown in the middle panel, where $S_{i}$ is the ith point of the faceness sigmoid, $S_{k}$ is the theoretical midpoint of the faceness sigmoid and $\min (A)$ is the minimum value of the ambiguity function. Right Panel: Group averaged reaction times during the face detection task. For all panels, error bars represent standard errors across subjects. B) Percent Signal change during event related tasks in all ROIs. In blue, the BOLD responses to the stimulus relevant, domain specific task, in red to the stimulus irrelevant task. For the majority of ROls, the stimulus relevant BOLD responses are larger across all phase levels relative to the stimulus irrelevant task. The green curve represents the differences between tasks. 
421

422

423

424

425 in Figure 3B.

426

427

428

429

430

431

432

433

434

435

436

437

438

439

440 effects with the current $\mathrm{N}=10$.

\section{Face and Fixation fMRI analyses}

\section{ANOVA Results}

The subjects mean percent signal change in response to each condition, for each ROI, is shown

The main effect of the task was significant $(p<0.05)$ in the IFFA $\left(F_{1,9}=20.559\right), \operatorname{rFFA}\left(F_{1,9}=\right.$ 13.131), IOFA $\left(F_{1,9}=48.595\right)$, rOFA $\left(F_{1,9}=14.304\right)$ and the $r S T S\left(F_{1,9}=9.182\right)$, with face detection driving larger BOLD responses relative to the fixation task.

$\left(F_{4,36}=13.136\right), \operatorname{rFFA}\left(F_{4,36}=35.567\right)$, IOFA $\left(F_{4,36}=7.56\right)$, rOFA $\left(F_{4,36}=22.866\right)$, ISTS $\left(F_{4,36}=7.98\right)$, rSTS $\left(F_{4,36}\right.$ $=5.637)$, the rAIT $\left(F_{4,36}=4.174\right)$. For these ROIs, paired sample post-hoc t-test $(p<.05$, corrected) showed that the $40 \%$ phase coherence is always significantly larger than $0 \%$.

There was a significant $(p<0.05)$ task by condition interaction term in the IFFA $\left(F_{4,36}=4.96\right)$, rFFA $\left(F_{4,36}=3.311\right)$, the rSTS $\left(F_{4,36}=3.620\right)$ and the rAIT $\left(F_{4,36}=3.84\right)$, indicating that amplitude increase during detection relatively to fixation was different for different phase coherence levels. Post-hoc t-tests carried out across tasks, within each condition revealed that for these ROIs only the $20 \%$ phase coherence conditions were always significantly ( $p<.05$ corrected) larger than all other conditions. The t-values (IFFA $(t(9)=4.407), r F F A(t(9)=4.132), r S T S(t(9)=4.684)$ and the $r A I T(t(9)=3.376))$ and related effect sizes (Hedges g*: IFFA: 1.361; rFFA: 1.795; rSTS: 1.791; and rAIT: 1.151) further indicate reliable and replicable 
444 during the face detection task relative to fixation in multiple core face processing areas. Specifically, we

445 found significant increases in functional connectivity between the 1) IFFA and the rFFA, right and IOFA,

446 rSTS and rAIT, 2) rFFA and rOFA, rSTS and rAIT, 3) the IOFA and the rSTS 4) the rOFA and the rSTS

447 (symmetrical connectivity between regions not repeated).

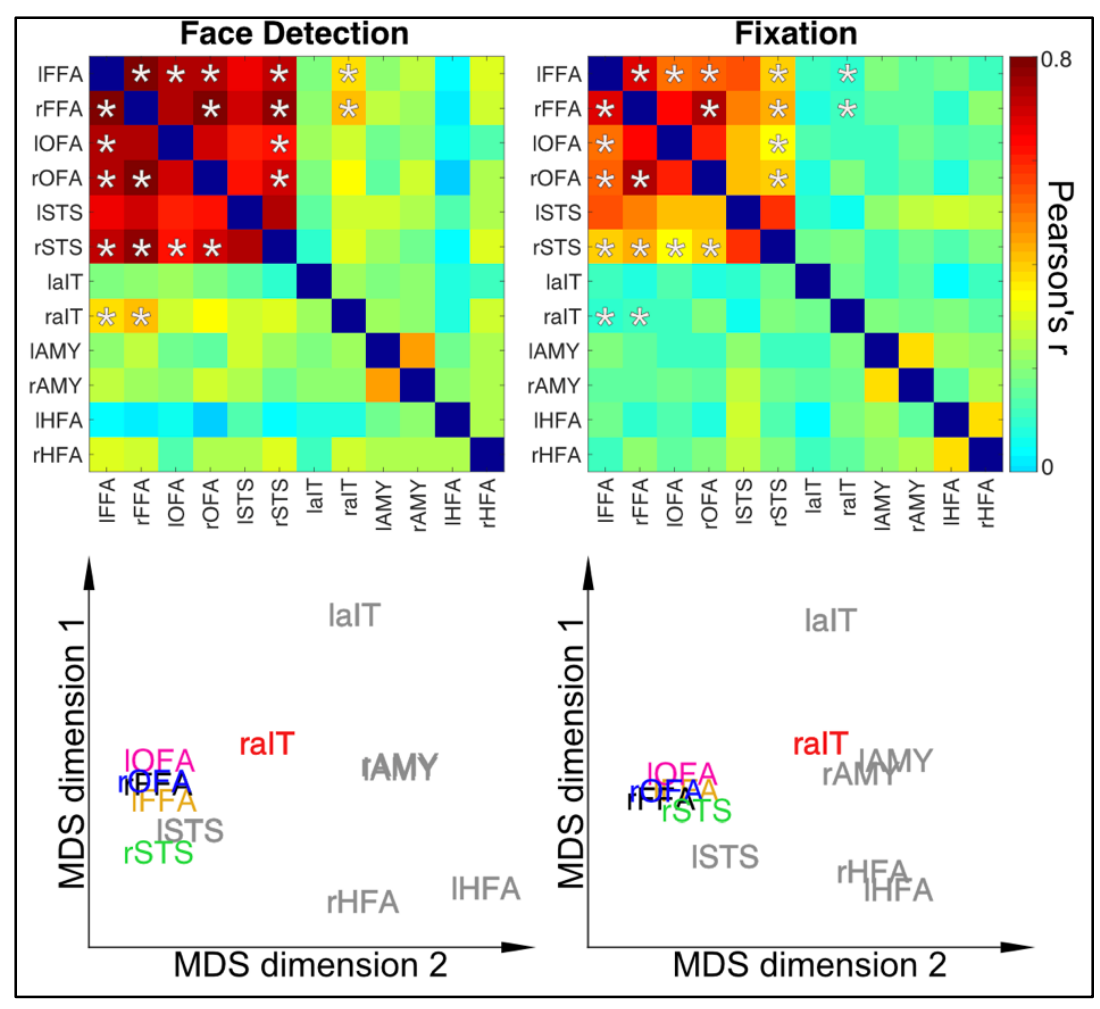

Figure 4. Dynamic Reconfiguration of Face Network as a function of Task Top Row: Connectivity Matrices for Stimulus Relevant Face Detection Task (Left) and Stimulus Irrelevant Fixation Task (Right). Asterisks indicate correlation coefficients that significantly $(p<0.05)$ difference between tasks and are identical between matrices for visualization purposes. Bottom Row. Classic Multidimensional Scaling for connectivity matrices highlights the higher proximity of the rAIT to the core face areas as a function of increased connectivity during face detection relative to the fixation task. ROls in grey text indicate those regions with no significant connectivity modulations across tasks.

Multidimensional scaling (MDS), which is a dimensionality reduction technique that allows 
bioRxiv preprint doi: https://doi.org/10.1101/2020.05.27.119677; this version posted November 17, 2020. The copyright holder for this preprint

(which was not certified by peer review) is the author/funder, who has granted bioRxiv a license to display the preprint in perpetuity. It is made available under aCC-BY-NC-ND 4.0 International license.

460 similarity of responses, highlights how the rAIT is more closely located to the FFAs (i.e. significantly more

461 correlated) during face detection relative to fixation (see Figure 4 Bottom; multidimensional space of

462 fixation rotated onto that of detection using Procrustes transformation).

463

464

Brain-Behavior Correlations

465

Figure 5A shows the correlation between the ambiguity score and the difference in the measured

brain activity between the stimulus-relevant (SR) and stimulus-irrelevant (SI) tasks. Correlations where

467 significant ( $p<0.05$, corrected) in the bilateral FFA and OFAs and the rAIT. 


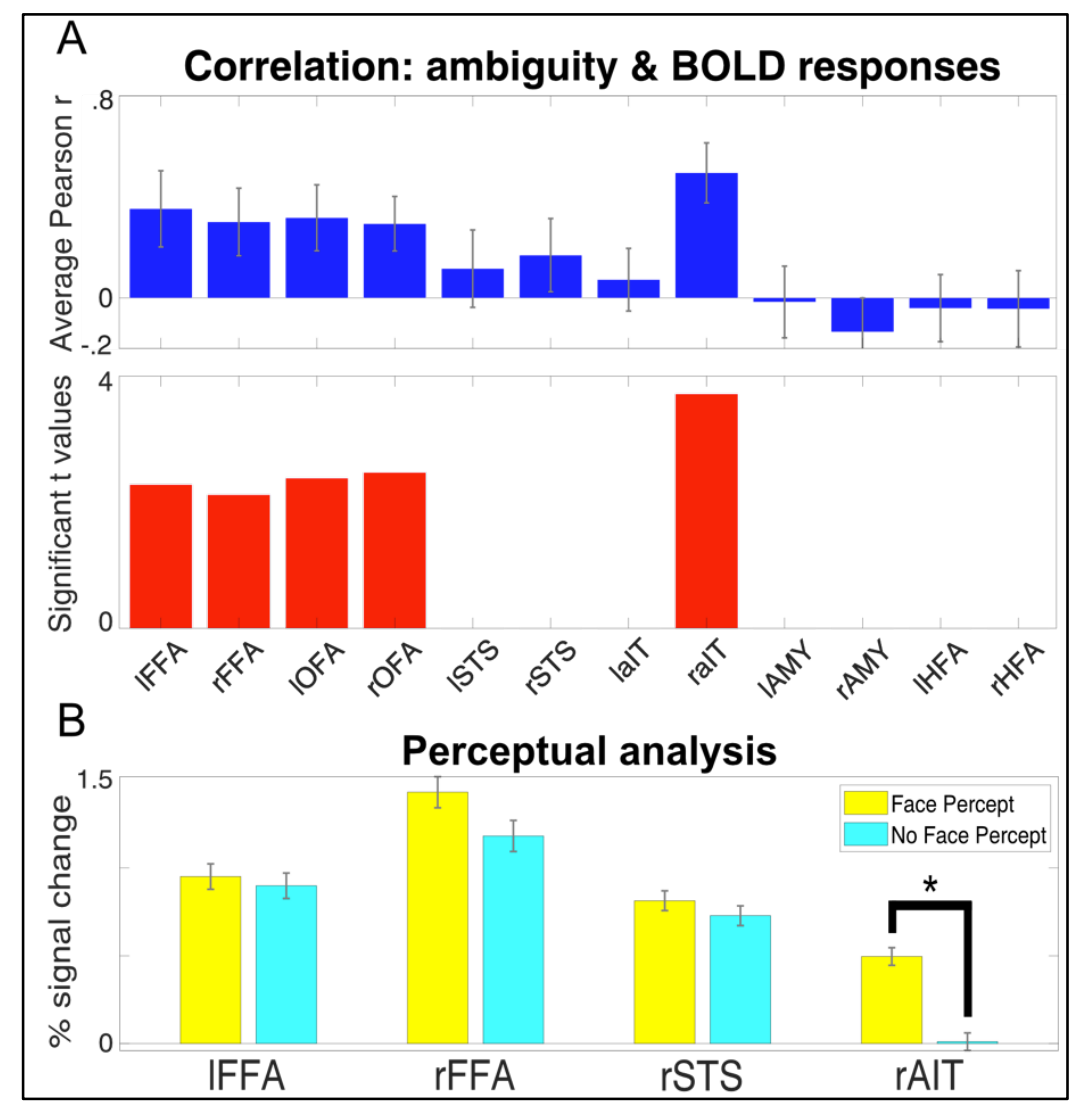

Figure 5. A) Brain-Behavior Correlations in FFAs, OFAs and rAIT. Top. The Pearson correlation coefficient between the ambiguity score and the difference in task BOLD responses. Bottom. For the regions reaching significance $(p<0.05)$, the left and right FFAs and OFAs, and $r A I T$, the t values are plotted. B) Average BOLD response to $20 \%$ phase coherence (i.e. the most ambiguous stimulus) for the trials in which participants reported a face percept (yellow) and no face percept (magenta). Responses are reported for the regions that showed a significant $(p<.05$ corrected) task by condition interaction. Star symbol indicates significantly $(p<.05$ corrected) difference in amplitude.

\section{Perceptual analysis}

For the $20 \%$ phase coherence condition only (i.e. the most ambiguous percept) we further separated the activity elicited by each trial according to participants perceptual response, creating 2 new conditions: "face percept" and "no face percept". We investigated amplitude differences between these 
bioRxiv preprint doi: https://doi.org/10.1101/2020.05.27.119677; this version posted November 17,2020 . The copyright holder for this preprint (which was not certified by peer review) is the author/funder, who has granted bioRxiv a license to display the preprint in perpetuity. It is made available under aCC-BY-NC-ND 4.0 International license.

485 corrected; see figure 5B). Moreover, paired sample t-tests contrasting the activation of each condition

486 for all ROIs against 0 further indicated significant ( $p<.05$ corrected) above baseline activation for all ROIs

487 and conditions, except for the "no face percept" response in the rAIT, which did not significantly differ

488 from baseline.

489

490 


\section{Discussion}

492

494

495

496

497

498

499

500

501

502

503

504

505

506

507

508

509

510

511

512

\section{Task Modulations \& Ambiguity}

Straightforward manipulation of task demands can produce changes in the amplitude of BOLD response in higher level areas, such as the FFA (Druzgal and D'Esposito, 2001; Kay and Yeatman, 2017; Vuilleumier et al., 2001; Wojciulik et al., 1998) or STS (Narumoto et al., 2001). Traditionally, however, these tasks manipulations are often indirectly related to the neural processing of the stimuli at hand, as they serve a more general purpose of directing attention towards (e.g. N-back) or away from the stimuli (e.g. fixation tasks, see Bokde et al., 2005; Druzgal and D’Esposito, 2001; Egner and Hirsch, 2005; Kay and Yeatman, 2017; Wojciulik et al., 1998). Changes in neural responses to identical visual inputs related to these unspecific changes in task demands often reveal broad contributions from attentional networks, including frontal and parietal regions (Szczepanski et al., 2013). While these manipulations can shed light upon the neural basis of general, non-specific top-down mechanisms, such as awareness, working memory demands or vigilance, they may fail to reveal fine-grained top-down modulations pertaining to the processing of a specific stimulus category. The approach used here instead builds on task manipulations that tap into a relevant stimulus dimension. This disambiguates the contributions of various regions within dedicated networks (here, the face processing network), by modulating the difficulty within a stimulus relevant task. In the context of this work, we will be referring to the modulatory forces that direct attention towards (e.g. N-Back) or away from (e.g. observe fixation) the stimuli as "non-specific top-down"; and to the task-specific modulations, as they pertain to task difficulty or cognitive load, as "domain-specific top-down". 
514 task is consistent with a large body of literature highlighting the conceptual and anatomical differences

515 between different types of attention. Posner and Peterson (1990) delineated 3 attention subsystems

516 devoted to orienting, detection, and alertness. Our results suggest that a design in which domain-

517 specificity difficulty is varied is capable of revealing the distinct modulation associated with different

518 attentional subsystems. The significant ( $p<.05$ corrected) main effect of task, indicates, in accordance

519 with previous reports (Druzgal and D'Esposito, 2001; Kay and Yeatman, 2017; Vuilleumier et al., 2001;

520 Wojciulik et al., 1998) that BOLD amplitude is on average larger during the stimulus relevant (SR) relative

521 to stimulus irrelevant (SI) task in canonical face processing areas: the bilateral FFA, OFA and the rSTS. In

522 line with previous work, (Druzgal and D’Esposito, 2001; Kay and Yeatman, 2017; Ress et al., 2000), we

523 suggest that these increases reflect broad top-down contributions relating to increased vigilance and

524 awareness of the stimuli, which have previously been shown to improve decoding performance (Dobs

525 et al., 2018).

By contrast, there are a specific subset of areas that show an interaction between task and

527 stimulus: the bilateral FFA as well as the rAIT and rSTS. Of these only the rAIT showed a significant

528 interaction, driven by the activity elicited by the $20 \%$ phase coherence images being larger during the SR

529 compared to the SI. This result indicates that, at least within the context of this work, this region's

530 responses are exclusively domain-specific top-down modulations. In this region in fact, other than for the

$53120 \%$ phase coherence, task demands do not alter BOLD amplitude to any other condition (Figure 3B).

533 of condition, driven by larger amplitude for the $40 \%$ phase relative to the $0 \%$ phase. As this comparison

534 is equivalent to a classical 'face vs scrambled face' linear contrast (Chen et al., 2007; Rossion et al., 2012) 
535 used to define stimulus selectivity, and consistent with the preferentiality of these regions for this

536 stimulus category, this result is unsurprising and won't be discussed further.

Post-hoc t-tests carried out on the significant task $\mathrm{x}$ condition interactions revealed that in all cortical regions exhibiting specific top-down modulations, the stimulus relevant task amplitude increases were always significant only for $20 \%$ phase coherence. Behaviorally, this condition was also found to be

542 the most perceptually ambiguous, i.e. the condition with the largest reported number of contrasting 543 percepts (i.e. "face" and "no face"). In the context of this study we mathematically defined ambiguity as 544 the inverse of the absolute difference from the inflection point of the sigmoid that describes the face 545 percept/detection behavioral responses (see Equation 1). Both extremes of the phase coherence 546 continuum therefore represent non-ambiguous percepts. That is, at low phase coherence (e.g. 0\%) 547 participants consistently reported no face percept and at high coherence (40\%) participants consistently 548 reported the presence of a face (Figure 3A). At the maximally ambiguous, $20 \%$ condition, the percept 549 was at its most unstable and thus most difficult to categorize, leading to a larger amplitude response. 550 This relative increase is consistent with prior work showing that task difficulty can modulate the BOLD 551 response not only in frontal or parietal regions (Culham et al., 2001; Gould et al., 2003) but also in these 552 category sensitive visual areas (Druzgal and D’Esposito, 2001). Notably, the reaction times measured in this study do not appear to capture this difficulty

554 increase (Figure 3A). This disconnect between reaction time and experimental performance has been 555 noted before in relation to accuracy and attentional cueing (van Ede et al., 2012) or to task difficulty 556 across a wide range of $\mathrm{N}$-back conditions (Lamichhane et al., 2020). In our data, this likely reflects a 
557 disconnect between the idea of "task difficulty" and "difficulty in categorization" which may provide

558 separate contributions to total reaction time.

\section{Top-down driven network reconfiguration}

562 thus the connectivity within specialized cortical networks (here, the face network), we compared

563 functional connectivity between tasks (see paragraph 4.6.2). By computing functional connectivity on

564 concatenated FIR curves, we: 1) discard the contribution of ongoing activity (which is not the focus of

565 this specific work); and 2) increase statistical power and thus the reliability of our correlational metric.

566 Broadly speaking, connectivity was significantly greater in core and extended regions (i.e. between FFAs,

567 OFAs and rSTS) during the stimulus relevant task, indicating greater communication among these areas,

568 presumably to fulfill task demands. In particular, we observed significantly ( $p<.05$ corrected) greater

569 connectivity between the rAIT and the FFAs during the face task relative to the fixation (Figure 4). A

570 number of studies have suggested a functional differentiation between the right and IFFA (Meng et al.,

571 2012; Rossion et al., 2000), with the former being more involced in holistic processing and the latter in

572 featural processing. It is therefore likely that the degraded stimuli used here (Figure 1) drive both

573 individual feature detection as well as holistic face processing; or that subjects flexibly adapt their

574 strategy depending on the available information, switching from featural (e.g. looking for eyes) to holistic

575 detection, thus engaging both FFAs.

577 can be recruited by the cortical face network to add an additional resource to resolve ambiguous 578 percepts. These results are consistent with the functional differentiation between core and extended 579 cortical face networks, according to which the core system mediates the representation of more basic 
580 aspects of face processing, while the extended system is involved in higher level cognitive functions and

581 can be selectively recruited to act in concert with the regions in the core system (Haxby et al., 2000).

of functional connectivity during task and at rest (Allen et al., 2014; Cribben et al., 2013; Debener et al.,

585 2006; Doucet et al., 2012; Hutchison et al., 2013; Sadaghiani et al., 2009) in response to cognitive and

586 behavioral demands (Gratton et al., 2018; Hutchison and Morton, 2016). Our results further expand

587 these views, indicating that even subtle changes in task demands, as those implemented here, can have

588 a dramatic impact over local network reconfigurations.

\section{$\underline{\text { Using Stimulus Ambiguity to Differentiate Functional Architecture }}$}

the source of top-down modulation. First, the correlation between the difference of the BOLD signal

between tasks, and each participant's behavior (ambiguity score) was only significant in the bilateral

FFAs and OFAs and the rAIT. By using task differences in BOLD responses to identical stimuli, we sought

595 to highlight task specific top-down effects. This finding, indicating correspondence between the

596 ambiguity function and the difference in BOLD amplitude across tasks within these ROIs, suggests that

597 this ambiguity signal originates from one of these regions. As the rAIT is further along the information

598 hierarchy, it is the plausible source of this signal. Moreover, functional connectivity analysis shows

599 increased connectivity between the rAIT and both FFAs, between OFAs and FFAs, but not between the

600 rAIT and either of the OFAs. In addition, the FFAs show both non-specific (significant main effect of task)

601 and domain-specific (significant task $x$ condition interaction) top-down effects, while the rAIT shows only

602 the task-specific, ambiguity related, top-down effects (significant interaction). 
these top-down signals when detecting objects such as faces or houses (Baldauf and Desimone, 2014;

Kay and Yeatman, 2017), these studies used indirect methods (i.e. 1-back; house or face) to examine

face detection. By manipulating difficulty within the context of face detection, we uncover a different,

607 within-network source of top-down modulation by directly stressing the face processing system. This

608 approach is similar to prior work finding modulation within the ventral temporal cortex when viewing

degraded facial stimuli (Fan et al., 2020).

Furthermore, there is evidence that the AIT is involved in resolving difficult stimuli in both

macaques and humans. For the former, prior work has implicated this region in learning ambiguous

612 stimulus rules related to concurrent discrimination (Bussey et al., 2002). Increases in ambiguity were

613 associated with worse performance in the macaques with perirhinal cortex (i.e. anterior inferior

614 temporal lobe) lesions. In humans, there is evidence that the AIT is associated with discriminating

615 individual face identities (Nasr and Tootell, 2012; Zhang et al., 2016), however these studies employed

616 tasks that did not necessarily tap directly into stimulus-specific dimensions. Though these studies offer

617 support for our findings, they are unable to disambiguate the relationship between behavior and

618 perception, as they either used a memory task, in which subjects could have used an image matching

619 strategy (Nasr and Tootell, 2012), or used only a fixation task (Zhang et al., 2016). Additional evidence

620 for the involvement of the AIT in ambiguous or difficult stimuli comes from outside the face perception

621 literature: this area is active when integrating conceptual and perceptual information (Martin et al.,

622 2018) and it is associated with identifying confusable objects (Clarke and Tyler, 2014; Tyler et al., 2013).

623 We therefore argue that the results presented here and those reported in previous work represent

624 indication that the ambiguity-related top-down signal during face detection originates in the rAIT, is fed

625 back to the FFAs and, from there, to the OFAs. Future work can explicitly assess this using specifically 
626 tailored research methods that provide better evidence of causality, such as depth dependent fMRI

627 analyses (Huber et al., 2017), intracranial EEG or fMRI with dynamic causal modeling (Friston et al., 2019).

628 in light of the low and inconsistent responses in IAIT, we advocate caution in interpreting these results.

646 Further work is therefore required to characterize the functional role of this and other areas (i.e. HFAs

647 and amygdalae) that displayed comparable responses. 
649 the bottom-up physical properties of the stimulus: regardless of the perceptual state, the $20 \%$ phase

650 coherence images always contained a face stimulus. Alternatively, this result can be due to the

651 contextual top-down induced by task demands (here a face detection task). That is, as tasks were blocked

652 by run, during SR runs, participants expected having to resolve perceptual judgment of an ambiguous

653 stimulus, and therefore looked for a face or a face feature in every trial. This is consistent with the

654 observation that, in the absence of a face, the FFA can be activated from contextual cues alone (Cox et

655 al., 2004).

657 have found amplitude differences in the FFA (Andrews and Schluppeck, 2004) or latency differences in

illusion also report that the FFA shows greater activation when faces are perceived (Andrews et al., 2002;

Hasson et al., 2001). With binocular rivalry, in which alternative images are shown to each eye, the FFA

661 also increases in activity when faces are perceived (Tong et al., 1998). In our present work we found that

662 the FFA did show a larger, however, non-significant increase when subjects reported seeing a face. This

663 apparent discrepancy with prior reports can be explained by differences in experimental paradigms.

664 Unlike Mooney faces, or face-related bistable stimuli, the $20 \%$ phase coherence stimuli used here always

665 have a physical face present. For binocular rivalry, the perceptual ambiguity is similar to bistable

666 perception, however binocular rivalry is ecologically implausible. The defining characteristic of these

667 probes is a dynamic switching amongst percepts despite identical visual input. Here, we have instead

668 focused on the ambiguity of the initial percept, which is more similar to approaches using degraded or

669 partially occluded static stimuli (Flounders et al., 2019; Frühholz et al., 2011). With our stimuli and

670 experimental manipulations, we isolate a unique and distinctive signal only in the rAlT that distinguishes

671 the subjective perception of a face. 
673 human primate research. Similar to humans, macaque monkeys possess a specialized cortical network

674 in inferotemporal cortex dedicated to processing faces (e.g. Tsao et al., 2003), however the exact correspondence between human and macaque face-selective areas is still unclear (Tsao et al., 2008a,

676 2006, 2003). While a degree of structural and functional correspondence has been achieved with regards

677 to the core regions (e.g. macaque middle face patches to human FFA (Rajimehr et al., 2009; Tsao et al., 678 2008a)), identifying a human homologue of the macaque anterior medial (AM) patch (the anterior most 679 patch) has been challenging. Tsao, Moeller, et al. (2008a) for example, failed to uncover a comparable 680 region in humans, attributing such failure to suceptibility related signal drop out due the putative 681 proximity of this area to the ear canal. Rajimehr and colleagues (Rajimehr et al., 2009) uncovered a face 682 specific region in human AIT in 5 out of 10 participants, but were unable to elucidate the nature of its 683 response properties. Here not only were we able to identify a human face selective region in AIT in all 684 subjects, but, importantly, we were able to define its response profile. Our results, linking the function 685 of the face AIT to subjective perception, are in line with recent electrophysiology reports, showing that 686 activity in the AM face patch is related to the animal's individual ability to detect a face (Moeller et al., 687 2017).

\section{Benefits of High Field Imaging}

As briefly mentioned in the above paragraph, much of the difficulty in imaging regions such as the AIT relates to low SNR associated because of susceptibility artifacts and inefficient RF transmit fields

692 in the ventral temporal lobes (Devlin et al., 2000). Moreover, areas such as the AIT are typically difficult 693 to image at more conventional (3T) field strengths due to low signal and are often not located in every 694 individual (e.g. $50 \%$ in Rajimehr et al., 2009), and often show large deviation from expected 
695 hemodynamic responses (e.g. Ramon et al., 2015). These issues can persist independent of field 696 strength.

Here we used UHF fMRI to capitalize on higher SNR, CNR, and acceleration performance maximizing $\mathrm{fMRI}$ sensitivity in these regions. It should be noted that moving to higher field alone is not sufficient to guarantee increased image quality in these regions. At UHF, while BOLD signal changes and

SNR do increase, BO and B1+ inhomogeneity also increase and have to be dealt with. Here the

701 combination of our experimental design, rigorous analytical approach, manual B0 shimming tailored to

702 all participants with a specific focus on anterior ventral temporal regions and flip angle optimizations

703 (see methods) yielded fruitful results. For all our participants we report consistent and corresponding

704 regions in the anterior inferior temporal cortex on both the left and right that preferentially respond to

705 faces and that are modulated by task demands. This increased sensitivity that led to large effect size and

706 ROI identification in all subjects, stems from a combination of parameters and sequence optimization.

707 Specifically, in addition to the increased SNR that accompanies UHF strength, unlike 3T acquisitions,

708 where no effort has been put forward to for optimizing B0 and flip angles, we manually adjusted B0

709 inhomogeneity and flip angles to maximize SNR for each subject. Moreover, relative to previous human

710 work, where functional voxels measured > $3 \mathrm{~mm}$ iso (e.g. (Rajimehr et al., 2009; Ramon et al., 2015)),

711 here, we used $1.6 \mathrm{~mm}$ iso voxels, minimizing partial volume effects and spin dephasing, ultimately

712 reducing signal loss in dropout regions (Thanh Vu et al., 2017). While the increased signal due to higher

713 field strengths can be a benefit, using high field alone is insufficient and can be, in fact, at times

714 detrimental. Appropriate consideration of tradeoffs, such as increased B0/B1+ instability, is necessary. 
718 orbital regions in macaques (Barat et al., 2018; Hadj-Bouziane et al., 2008; Tsao et al., 2008b). However,

719 evidence for the existence of these areas in humans has been mixed, uncovering such regions in

720 approximately half (Troiani et al., 2016) or one-third of the study population (Troiani et al., 2019; Tsao

721 et al., 2008a). In the current work, we are able to identify these areas in all subjects during the localizer

722 portion of the study. Activation was proximal to the H-Shaped sulci, consistent with both prior reports

723 (Troiani et al., 2019) and across subjects, as visualized after normalization to MNI space (Figure 2). Like

724 the other ROIs in the face preferential cortical network, these regions exhibited positive responses to

725 faces and significantly $(p<.05)$ greater activity to faces relative to scrambled stimuli or objects (See 726 Methods).

Although we did not observe meaningful stimulus relevant task modulations we did observe an

729 during face detection: the rHFA exhibited a non-significant increase in activation, while its left

730 counterpart exhibited a non-significant decrease. These results should be interpreted with caution, as

731 the primary tasks produced low response amplitudes relative to the localizer. The absence of strong

732 activation in the HFAs, as well as other regions such as the amygdalae and left AIT, during the primary

733 task despite prominent responses in the localizer task is likely due to a number of differences between

734 the tasks. These include, but are not limited to, difference in presentation timings (i.e. 12 secs vs. 2 secs

735 on/off) and the fact that the localizer used stimulus presentations with non-degraded, full faces with

736 variable expressions and gaze directions.

That these task differences drove larger responses during the localizer task in the HFAs is 738 congruent with prior findings, namely that these areas have been suggested to be involved in social and 739 emotional aspects of face processing in non-human primates (Barat et al., 2018), aspects that, within the 
740 primary task context, are irrelevant. Our failure to find task modulations is therefore consistent with the

741 more complex evaluative role of the frontal cortices (Noonan et al., 2012) and its engagement in social

742 perception tasks (Barat et al., 2018; Beer et al., 2006; Freeman et al., 2010; Mah et al., 2004).

744 frontal areas in humans, it will be essential to perform further research that build on these differences

745 and further manipulate context, value and/or salience in order to elucidate the functional role of regions

746 such as the HFAs during face perception and processing. 


\section{Conclusion}

750 this study we combined high SNR fMRI with domain-specific attention and observed 2 types of top-down

751 scaling mechanisms: 1) a broad gain effect related to drawing attention to the stimuli, manifesting as an

752 unspecific amplitude increase across conditions; and 2) an additional scaling of specific conditions

753 dictated by domain-specific task requirements. We explain the latter in terms of perceptual ambiguity

754 and suggest that the ambiguity signal originates in the rAIT. Importantly, only in the rAIT is both

755 preferentially active under challenging conditions and predictive of the subjects' perceptual judgments.

756 We further show that subtle changes in task demands can lead to dramatic changes in network

757 reconfigurations. Our results suggest that the combination of an explicit face detection and stimulus

758 matched control task with low noise fMRI capable of resolving previously inaccessible regions of the

759 human brain may be the only way to understand the changes underlying human cognitive flexibility 
bioRxiv preprint doi: https://doi.org/10.1101/2020.05.27.119677; this version posted November 17, 2020. The copyright holder for this preprint (which was not certified by peer review) is the author/funder, who has granted bioRxiv a license to display the preprint in perpetuity. It is made available under aCC-BY-NC-ND 4.0 International license.

\section{Acknowledgments}

763 Funding for this study was provided by National Institutes of Health Grants RF1 MH117015

764 (Ghose), RF1 MH116978 (Yacoub), and P30 NS076408 (Ugurbil). The authors would also like to thank Dr.

765 Kendrick Kay for conceptual discussions regarding stimulus ambiguity and feedback.

766

767 
bioRxiv preprint doi: https://doi.org/10.1101/2020.05.27.119677; this version posted November 17,2020 . The copyright holder for this preprint (which was not certified by peer review) is the author/funder, who has granted bioRxiv a license to display the preprint in perpetuity. It is made available under aCC-BY-NC-ND 4.0 International license.

\section{Competing Interests}




\section{References}

771

772 Alexander GE, DeLong MR, Strick PL. 1986. Parallel Organization of Functionally Segregated Circuits

773

774

Linking Basal Ganglia and Cortex. Annu Rev Neurosci 9:357-381. doi:10.1146/annurev.ne.09.030186.002041

775

Allen EA, Damaraju E, Plis SM, Erhardt EB, Eichele T, Calhoun VD. 2014. Tracking whole-brain connectivity dynamics in the resting state. Cereb Cortex 24:663-676. doi:10.1093/cercor/bhs352

777

778

779

780

781

782

783

784

785

786

787

Andrews TJ, Schluppeck D. 2004. Neural responses to Mooney images reveal a modular representation of faces in human visual cortex. Neuroimage 21:91-98. doi:10.1016/j.neuroimage.2003.08.023

Andrews TJ, Schluppeck D, Homfray D, Matthews P, Blakemore C. 2002. Activity in the Fusiform Gyrus Predicts Conscious Perception of Rubin's Vase-Face Illusion. Neuroimage 17:890-901. doi:10.1006/nimg.2002.1243

Arcaro MJ, Livingstone MS. 2017. Retinotopic organization of scene areas in macaque inferior temporal cortex. J Neurosci 37:7373-7389. doi:10.1523/JNEUROSCI.0569-17.2017

Baldauf D, Desimone R. 2014. Neural mechanisms of object-based attention. Science (80- ) 344:424-427. doi:10.1126/science.1247003

Barat E, Wirth S, Duhamel JR. 2018. Face cells in orbitofrontal cortex represent social categories. Proc Natl Acad Sci U S A 115:E11158-E11167. doi:10.1073/pnas.1806165115

Bassett DS, Wymbs NF, Porter MA, Mucha PJ, Carlson JM, Grafton ST. 2011. Dynamic reconfiguration of human brain networks during learning. Proc Natl Acad Sci U S A 108:7641-7646. doi:10.1073/pnas.1018985108 
791 Beer JS, John OP, Scabini D, Knight RT. 2006. Orbitofrontal cortex and social behavior: Integrating selfmonitoring and emotion-cognition interactions. J Cogn Neurosci 18:871-879.

Bokde ALW, Dong W, Born C, Leinsinger G, Meindl T, Teipel SJ, Reiser M, Hampel H. 2005. Task difficulty in a simultaneous face matching task modulates activity in face fusiform area. Cogn Brain Res doi:10.1162/jocn.2006.18.6.871

Bussey TJ, Saksida LM, Murray EA. 2002. Perirhinal cortex resolves feature ambiguity in complex visual discriminations. Eur J Neurosci 15:365-374. doi:10.1046/j.0953-816x.2001.01851.x

Chen CC, Kao KLC, Tyler CW. 2007. Face configuration processing in the human brain: The role of symmetry. Cereb Cortex 17:1423-1432. doi:10.1093/cercor/bhl054

Chen NK, Dickey CC, Yoo SS, Guttmann CRG, Panych LP. 2003. Selection of voxel size and slice orientation for $f M R I$ in the presence of susceptibility field gradients: Application to imaging of the amygdala. Neuroimage 19:817-825. doi:10.1016/S1053-8119(03)00091-0

Clarke A, Tyler LK. 2014. Object-specific semantic coding in human perirhinal cortex. J Neurosci 34:4766-

Cohen MR, Maunsell JHR. 2011. Using Neuronal Populations to Study the Mechanisms Underlying Spatial and Feature Attention. Neuron 70:1192-1204. doi:10.1016/j.neuron.2011.04.029

Cox D, Meyers E, Sinha P. 2004. Contextually Evoked Object-Specific Responses in Human Visual Cortex. Science (80- ) 304:115-117. doi:10.1126/science.1093110 neuroimages. Comput Biomed Res 29:162-173. doi:10.1006/cbmr.1996.0014 
812 Cribben I, Wager TD, Lindquist MA. 2013. Detecting functional connectivity change points for singlesubject fMRI data. Front Comput Neurosci 7. doi:10.3389/fncom.2013.00143

814 Culham JC, Cavanagh P, Kanwisher NG. 2001. Attention response functions: Characterizing brain areas using fMRI activation during parametric variations of attentional load. Neuron 32:737-745.

817 Cumming G. 2012. Understanding the new statistics: Effect sizes, confidence intervals, and metadoi:10.1016/S0896-6273(01)00499-8

Dale AM, Fischl B, Sereno MI. 1999. Cortical Surface-Based Analysis. Neuroimage 9:179-194. doi:10.1006/nimg.1998.0395

\section{2}

823

824

825

826

827

828

829

830

831

832

De Martino F, Moerel M, Ugurbil K, Goebel R, Yacoub E, Formisano E. 2015. Frequency preference and attention effects across cortical depths in the human primary auditory cortex. Proc Natl Acad Sci U S A 112:16036-16041. doi:10.1073/pnas.1507552112

Debener S, Ullsperger M, Siegel M, Engel AK. 2006. Single-trial EEG-fMRI reveals the dynamics of cognitive function. Trends Cogn Sci 10:558-563. doi:10.1016/j.tics.2006.09.010

Desimone R, Albright TD, Gross CG, Bruce C. 1984. Stimulus-selective properties of inferior temporal neurons in the macaque. J Neurosci 4:2051-2062. doi:10.1523/jneurosci.04-08-02051.1984

Devlin JT, Russell RP, Davis MH, Price CJ, Wilson J, Moss HE, Matthews PM, Tyler LK. 2000. Susceptibilityinduced loss of signal: Comparing PET and fMRI on a semantic task. Neuroimage 11:589-600. doi:10.1006/nimg.2000.0595

Dobs K, Schultz J, Bülthoff I, Gardner JL. 2018. Task-dependent enhancement of facial expression and 
identity representations in human cortex. Neuroimage

172:689-702.

$$
\text { doi:10.1016/j.neuroimage.2018.02.013 }
$$

835

836

837

838

839

840

841

842

843

Fischl B, Liu A, Dale AM. 2001. Automated manifold surgery: Constructing geometrically accurate and

Doucet G, Naveau M, Petit L, Zago L, Crivello F, Jobard G, Delcroix N, Mellet E, Tzourio-Mazoyer N, Mazoyer B, Joliot M. 2012. Patterns of hemodynamic low-frequency oscillations in the brain are modulated by the nature of free thought during rest. Neuroimage 59:3194-3200. doi:10.1016/j.neuroimage.2011.11.059

Druzgal TJ, D’Esposito M. 2003. Dissecting contributions of prefrontal cortex and fusiform face area to face working memory. J Cogn Neurosci 15:771-784. doi:10.1162/089892903322370708

Druzgal TJ, D’Esposito M. 2001. Activity in fusiform face area modulated as a function of working memory load. Cogn Brain Res 10:355-364. doi:10.1016/S0926-6410(00)00056-2

Egner T, Hirsch J. 2005. Cognitive control mechanisms resolve conflict through cortical amplification of task-relevant information. Nat Neurosci 8:1784-1790. doi:10.1038/nn1594

Fan X, Wang F, Shao H, Zhang P, He S. 2020. The bottom-up and top-down processing of faces in the human occipitotemporal cortex. Elife 9. doi:10.7554/eLife.48764

Farzaneh F, Riederer SJ, Pelc NJ. 1990. Analysis of T2 limitations and off-resonance effects on spatial resolution and artifacts in echo-planar imaging. Magn Reson Med 14:123-139. doi:10.1002/mrm.1910140112

Felleman DJ, Van Essen DC. 1991. Distributed hierarchical processing in the primate cerebral cortex. Cereb Cortex 1:1-47. doi:10.1093/cercor/1.1.1 topologically correct models of the human cerebral cortex. IEEE Trans Med Imaging 20:70-80. 
Fischl B, Salat DH, Busa E, Albert M, Dieterich M, Haselgrove C, Van Der Kouwe A, Killiany R, Kennedy D, Klaveness S, Montillo A, Makris N, Rosen B, Dale AM. 2002. Whole brain segmentation: Automated labeling of neuroanatomical structures in the human brain. Neuron 33:341-355.

Fischl B, Van Der Kouwe A, Destrieux C, Halgren E, Ségonne F, Salat DH, Busa E, Seidman LJ, Goldstein J,

Fisher RA. 1915. Frequency Distribution of the Values of the Correlation Coefficient in Samples from an Indefinitely Large Population. Biometrika 10:507. doi:10.2307/2331838

Flounders MW, González-García C, Hardstone R, He BJ. 2019. Neural dynamics of visual ambiguity resolution by perceptual prior. Elife 8. doi:10.7554/eLife.41861

Freeman JB, Rule NO, Adams RB, Ambady N. 2010. The neural basis of categorical face perception: Graded representations of face gender in fusiform and orbitofrontal cortices. Cereb Cortex

Freeman PR, Hedges L V., Olkin I, Freeman PR, Hedges L V., Olkin I, Rosenbaum PR, Hedges L V., Olkin I, 20:1314-1322. doi:10.1093/cercor/bhp195 Freeman PR, Hedges L V., Olkin I. 1986. Statistical Methods for Meta-Analysis., Biometrics. Academic Press. doi:10.2307/2531069 revisited. Neuroimage 199:730-744. doi:10.1016/j.neuroimage.2017.02.045 
875

876

\section{7}

878

879

880

881

\section{2}

883

884

885

886

887

888

889

890

891

892

893

894

895 Gratton C, Laumann TO, Nielsen AN, Gree
AZ, Schlaggar BL, Dosenbach NUF, P
Stable Group and Individual Factors
doi:10.1016/j.neuron.2018.03.035
Grill-Spector K, Weiner KS. 2014. The funct
in categorization. Nat Rev Neurosci
Hadj-Bouziane F, Bell AH, Knusten TA,
expressions is independent of face
Sci U S A 105:5591-5596. doi:10.10
doi:10.1016/j.neuroimage.2003.11.
Handwerker DA, Ollinger JM, D'Esposito
subjects and brain regions and the

Gonzalez-Castillo J, Bandettini PA. 2018. Task-based dynamic functional connectivity: Recent findings and open questions. Neuroimage 180:526-533. doi:10.1016/j.neuroimage.2017.08.006

Gould RL, Brown RG, Owen AM, Ffytche DH, Howard RJ. 2003. fMRI BOLD response to increasing task difficulty during successful paired associates learning. Neuroimage 20:1006-1019. doi:10.1016/S1053-8119(03)00365-3

Gratton C, Laumann TO, Nielsen AN, Greene DJ, Gordon EM, Gilmore AW, Nelson SM, Coalson RS, Snyder AZ, Schlaggar BL, Dosenbach NUF, Petersen SE. 2018. Functional Brain Networks Are Dominated by Stable Group and Individual Factors, Not Cognitive or Daily Variation. Neuron 98:439-452.e5.

Grill-Spector K, Weiner KS. 2014. The functional architecture of the ventral temporal cortex and its role in categorization. Nat Rev Neurosci 15:536-548. doi:10.1038/nrn3747

Hadj-Bouziane F, Bell AH, Knusten TA, Ungerleider LG, Tootell RBH. 2008. Perception of emotional expressions is independent of face selectivity in monkey inferior temporal cortex. Proc Natl Acad Sci U S A 105:5591-5596. doi:10.1073/pnas.0800489105

Hasson U, Hendler T, Bashat D Ben, Malach R. 2001. Vase or face? A neural correlate of shape-selective grouping processes in the human brain. J Cogn Neurosci 13:744-753. doi:10.1162/08989290152541412 
897 Haxby J V., Gobbini MI, Furey ML, Ishai A, Schouten JL, Pietrini P. 2001. Distributed and overlapping representations of faces and objects in ventral temporal cortex. Science (80- ) 293:2425-2430.

Haxby J V., Hoffman EA, Gobbini MI. 2000. The distributed human neural system for face perception.

\section{2}

903

904

905

906

907

908

909

910

911

912

\section{3}

914

915

916

917

Trends Cogn Sci 4:223-233. doi:10.1016/S1364-6613(00)01482-0

Hedges L V. 1981. Distribution Theory for Glass's Estimator of Effect Size and Related Estimators. J Educ Stat 6:107. doi:10.2307/1164588

Hubel DH, Wiesel TN. 1959. Receptive fields of single neurones in the cat's striate cortex. J Physiol 148:574-591. doi:10.1113/jphysiol.1959.sp006308

Huber L, Handwerker DA, Jangraw DC, Chen G, Hall A, Stüber C, Gonzalez-Castillo J, Ivanov D, Marrett S, Guidi M, Goense J, Poser BA, Bandettini PA. 2017. High-Resolution CBV-fMRI Allows Mapping of Laminar Activity and Connectivity of Cortical Input and Output in Human M1. Neuron 96:12531263.e7. doi:10.1016/j.neuron.2017.11.005

Hutchison RM, Morton JB. 2016. It's a matter of time: Reframing the development of cognitive control as a modification of the brain's temporal dynamics. Dev Cogn Neurosci, Flux Congress 2014 18:7077. doi:10.1016/j.den.2015.08.006

Hutchison RM, Womelsdorf T, Gati JS, Everling S, Menon RS. 2013. Resting-state networks show dynamic functional connectivity in awake humans and anesthetized macaques. Hum Brain Mapp 34:21542177. doi:10.1002/hbm.22058

Kabbara A, EL Falou W, Khalil M, Wendling F, Hassan M. 2017. The dynamic functional core network of the human brain at rest. Sci Rep 7:2936. doi:10.1038/s41598-017-03420-6 
918 Kanwisher N, McDermott J, Chun MM. 1997. The fusiform face area: A module in human extrastriate cortex specialized for face perception. J Neurosci 17:4302-4311. doi:10.1523/jneurosci.17-11-

Kanwisher N, Yovel G. 2006. The fusiform face area: A cortical region specialized for the perception of faces. Philos Trans R Soc B Biol Sci 361:2109-2128. doi:10.1098/rstb.2006.1934

\section{3}

924

925

926

927

928

929

930

931

932

933

934

935

936

937

938 04302.1997

Kay KN, Yeatman JD. 2017. Bottom-up and top-down computations in word- and face-selective cortex. Elife 6:e22341. doi:10.7554/eLife.22341

Lakens D. 2013. Calculating and reporting effect sizes to facilitate cumulative science: A practical primer for t-tests and ANOVAs. Front Psychol 4:863. doi:10.3389/fpsyg.2013.00863

Lamichhane B, Westbrook A, Cole MW, Braver TS. 2020. Exploring brain-behavior relationships in the Nback task. Neuroimage 212:116683. doi:10.1016/j.neuroimage.2020.116683

Laughlin SB, Sejnowski TJ. 2003. Communication in neuronal networks. Science (80- ) 301:1870-1874. doi:10.1126/science.1089662

Leung AWS, Alain C. 2011. Working memory load modulates the auditory "What" and "Where" neural networks. Neuroimage 55:1260-1269. doi:10.1016/j.neuroimage.2010.12.055

Lewis LD, Setsompop K, Rosen BR, Polimeni JR. 2018. Stimulus-dependent hemodynamic response timing across the human subcortical-cortical visual pathway identified through high spatiotemporal resolution 7T fMRI. Neuroimage 181:279-291. doi:10.1016/j.neuroimage.2018.06.056

Li Q, Hill Z, He BJ. 2014. Spatiotemporal dissociation of brain activity underlying subjective awareness, objective performance and confidence. J Neurosci 34:4382-4395. doi:10.1523/JNEUROSCI.182013.2014 
939 Li X, Morgan PS, Ashburner J, Smith J, Rorden C. 2016. The first step for neuroimaging data analysis: DICOM to NIfTI conversion. J Neurosci Methods 264:47-56. doi:10.1016/j.jneumeth.2016.03.001

941 Liu B, Zhu T, Zhong J. 2015. Comparison of quality control software tools for diffusion tensor imaging. Magn Reson Imaging 33:276-285. doi:10.1016/j.mri.2014.10.011

943

944

\section{5}

946

947

948

949

950

951

952

953

954

955

956

957

\section{8}

959

Mah L, Arnold MC, Grafman J. 2004. Impairment of social perception associated with lesions of the prefrontal cortex. Am J Psychiatry 161:1247-1255. doi:10.1176/appi.ajp.161.7.1247

Martin CB, Douglas D, Newsome RN, Man LLY, Barense MD. 2018. Integrative and distinctive coding of visual and conceptual object features in the ventral visual stream. Elife 7:e31873. doi:10.7554/eLife.31873

Martinez-Trujillo JC, Treue S. 2004. Feature-based attention increases the selectivity of population responses in primate visual cortex. Curr Biol 14:744-751. doi:10.1016/j.cub.2004.04.028

McCarthy G, Puce A, Gore JC, Allison T. 1997. Face-specific processing in the human fusiform gyrus. J Cogn Neurosci 9:605-610. doi:10.1162/jocn.1997.9.5.605

Meng M, Cherian T, Singal G, Sinha P. 2012. Lateralization of face processing in the human brain. Proc $R$ Soc B Biol Sci 279:2052-2061. doi:10.1098/rspb.2011.1784

Moeller S, Crapse T, Chang L, Tsao DY. 2017. The effect of face patch microstimulation on perception of faces and objects. Nat Neurosci 20:743-752. doi:10.1038/nn.4527

Moeller S, Freiwald WA, Tsao DY. 2008. Patches with links: A unified system for processing faces in the macaque temporal lobe. Science (80- ) 320:1355-1359. doi:10.1126/science.1157436

Mooney CM. 1957. Age in the development of closure ability in children. Can J Psychol 11:219-226. doi:10.1037/h0083717 
960 Narumoto J, Okada T, Sadato N, Fukui K, Yonekura Y. 2001. Attention to emotion modulates fMRI activity

in human right superior temporal sulcus. Cogn Brain Res 12:225-231. doi:10.1016/S0926-

963

964

965

966

967

968

969

970

971

972

973

974

975

976

977

978

979

980

6410(01)00053-2

Nasr S, Tootell RBH. 2012. Role of fusiform and anterior temporal cortical areas in facial recognition. Neuroimage 63:1743-1753. doi:10.1016/j.neuroimage.2012.08.031

Noonan MP, Kolling N, Walton ME, Rushworth MFS. 2012. Re-evaluating the role of the orbitofrontal cortex in reward and reinforcement. Eur J Neurosci 35:997-1010. doi:10.1111/j.14609568.2012.08023.x

Olman CA, Davachi L, Inati S. 2009. Distortion and signal loss in medial temporal lobe. PLoS One 4. doi:10.1371/journal.pone.0008160

Parker AJ, Newsome WT. 1998. SENSE AND THE SINGLE NEURON: Probing the Physiology of Perception. Annu Rev Neurosci 21:227-277. doi:10.1146/annurev.neuro.21.1.227

Perrett DI, Rolls ET, Caan W. 1982. Visual neurones responsive to faces in the monkey temporal cortex. Exp Brain Res 47:329-342. doi:10.1007/BF00239352

Pessoa L, Gutierrez E, Bandettini P, Ungerleider L. 2002. Neural correlates of visual working memory: fMRI amplitude predicts task performance. Neuron 35:975-987. doi:10.1016/S08966273(02)00817-6

Posner MI, Petersen SE. 1990. The Attention System of the Human Brain. Annu Rev Neurosci 13:25-42. doi:10.1146/annurev.ne.13.030190.000325

Price AR, Bonner MF, Peelle JE, Grossman M. 2017. Neural coding of fine-grained object knowledge in perirhinal cortex. bioRxiv 194829:1-18. doi:doi.org/10.1101/194829 
981 Rajimehr R, Young JC, Tootell RBH. 2009. An anterior temporal face patch in human cortex, predicted by macaque maps. Proc Natl Acad Sci U S A 106:1995-2000. doi:10.1073/pnas.0807304106

983

984

985

986

987

988

989

990

991

992

993

994

995

996

997

998

999

1000

1001

Ramon M, Vizioli L, Liu-Shuang J, Rossion B. 2015. Neural microgenesis of personally familiar face recognition. Proc Natl Acad Sci U S A 112:E4835-E4844. doi:10.1073/pnas.1414929112

Ress D, Backus BT, Heeger DJ. 2000. Activity in primary visual cortex predicts performance in a visual detection task. Nat Neurosci 3:940-945. doi:10.1038/78856

Rossion B, Dricot L, Devolder A, Bodart JM, Crommelinck M, De Gelder B, Zoontjes R, Gelder B De, Zoontjes R. 2000. Hemispheric asymmetries for whole-based and part-based face processing in the human fusiform gyrus. J Cogn Neurosci 12:793-802. doi:10.1162/089892900562606

Rossion B, Hanseeuw B, Dricot L. 2012. Defining face perception areas in the human brain: A large-scale factorial fMRI face localizer analysis. Brain Cogn 79:138-157. doi:10.1016/j.bandc.2012.01.001

Saad ZS, Reynolds RC. 2012. SUMA. Neuroimage 62:768-773. doi:10.1016/j.neuroimage.2011.09.016

Sadaghiani S, Hesselmann G, Kleinschmidt A. 2009. Distributed and antagonistic contributions of ongoing activity fluctuations to auditory stimulus detection. J Neurosci 29:13410-13417. doi:10.1523/JNEUROSCI.2592-09.2009

Smith SM, Beckmann CF, Andersson J, Auerbach EJ, Bijsterbosch J, Douaud G, Duff E, Feinberg DA, Griffanti L, Harms MP, Kelly M, Laumann T, Miller KL, Moeller S, Petersen S, Power J, SalimiKhorshidi G, Snyder AZ, Vu AT, Woolrich MW, Xu J, Yacoub E, Uğurbil K, Van Essen DC, Glasser MF. 2013. Resting-state $\mathrm{fMRI}$ in the Human Connectome Project. Neuroimage, Mapping the Connectome 80:144-168. doi:10.1016/j.neuroimage.2013.05.039

Stigliani A, Weiner KS, Grill-Spector K. 2015. Temporal processing capacity in high-level visual cortex is 
Szczepanski SM, Pinsk MA, Douglas MM, Kastner S, Saalmann YB. 2013. Functional and structural architecture of the human dorsal frontoparietal attention network. Proc Natl Acad Sci U S A 110:15806-15811. doi:10.1073/pnas.1313903110

D. 2018. Characterization of the hemodynamic response function across the

$$
\text { majority }
$$$$
\text { of }
$$

human

cerebral

cortex.

Neuroimage

173:322-331. doi:10.1016/j.neuroimage.2018.02.061

Thanh Vu A, Jamison K, Glasser MF, Smith SM, Coalson T, Moeller S, Auerbach EJ, Uğurbil K, Yacoub E. 2017. Tradeoffs in pushing the spatial resolution of fMRI for the 7T Human Connectome Project. Neuroimage, Cleaning up the fMRI time series: Mitigating noise with advanced acquisition and correction strategies 154:23-32. doi:10.1016/j.neuroimage.2016.11.049

Troiani V, Dougherty CC, Michael AM, Olson IR. 2016. Characterization of face-selective patches in orbitofrontal cortex. Front Hum Neurosci 10:1-14. doi:10.3389/fnhum.2016.00279

Troiani V, Patti MA, Adamson K. 2019. The use of the orbitofrontal H-sulcus as a reference frame for value signals. Eur J Neurosci n/a:ejn.14590. doi:10.1111/ejn.14590

Tsao DY, Freiwald WA, Knutsen TA, Mandeville JB, Tootell RBH. 2003. Faces and objects in macaque cerebral cortex. Nat Neurosci 6:989-995. doi:10.1038/nn1111

1021 Tsao DY, Freiwald WA, Tootell RBH, Livingstone MS. 2006. A cortical region consisting entirely of faceselective cells. Science (80- ) 311:670-674. doi:10.1126/science.1119983 
1023 Tsao DY, Moeller S, Freiwald WA. 2008a. Comparing face patch systems in macaques and humans. Proc

Natl Acad Sci U S A 105:19514-19519. doi:10.1073/pnas.0809662105

1025 Tsao DY, Schweers N, Moeller S, Freiwald WA. 2008b. Patches of face-selective cortex in the macaque

1026 frontal lobe. Nat Neurosci 11:877-879. doi:10.1038/nn.2158

1027 Tyler LK, Chiu S, Zhuang J, Randall B, Devereux BJ, Wright P, Clarke A, Taylor KI. 2013. Objects and 1028 categories: Feature statistics and object processing in the ventral stream. J Cogn Neurosci 25:17231735. doi:10.1162/jocn_a_00419

1030

1031

1032

1033

1034

1035

1036

1037

1038

1039

1040

1041

1042

1043

van Ede F, de Lange FP, Maris E. 2012. Attentional cues affect accuracy and reaction time via different cognitive and neural processes. J Neurosci 32:10408-10412. doi:10.1523/JNEUROSCI.1337-12.2012

Vogels R, Orban GA. 1994. Activity of inferior temporal neurons during orientation discrimination with successively presented gratings. J Neurophysiol 71:1428-1451. doi:10.1152/jn.1994.71.4.1428

Vuilleumier P, Armony JL, Driver J, Dolan RJ. 2001. Effects of attention and emotion on face processing in the human brain: An event-related fMRI study. Neuron 30:829-841. doi:10.1016/S08966273(01)00328-2

Wang SSH, Shultz JR, Burish MJ, Harrison KH, Hof PR, Towns LC, Wagers MW, Wyatt KD. 2008. Functional trade-offs in white matter axonal scaling. J Neurosci 28:4047-4056. doi:10.1523/JNEUROSCI.555905.2008

Wilcox R. 2005. Introduction to Robust Estimation and Hypothesis Testing, Technometrics. Academic Press. doi:10.1198/tech.2005.s334

Willenbockel V, Sadr J, Fiset D, Horne GO, Gosselin F, Tanaka JW. 2010. Controlling low-level image properties: The SHINE toolbox. Behav Res Methods 42:671-684. doi:10.3758/BRM.42.3.671 
1044 Wojciulik E, Kanwisher N, Driver J. 1998. Covert visual attention modulates face-specific activity in the

1045 human fusiform gyrus: fMRI study. J Neurophysiol 79:1574-1578. doi:10.1152/jn.1998.79.3.1574

1046 Young IR, Cox IJ, Bryant DJ, Bydder GM. 1988. The benefits of increasing spatial resolution as a means of 1047 reducing artifacts due to field inhomogeneities. Magn Reson Imaging 6:585-590. 1048 doi:10.1016/0730-725X(88)90133-6

1049 Zhang H, Japee S, Nolan R, Chu C, Liu N, Ungerleider LG. 2016. Face-selective regions differ in their ability 1050 to classify facial expressions. Neuroimage 130:77-90. doi:10.1016/j.neuroimage.2016.01.045

1051 Zhang RY, Kay K. 2020. Flexible top-down modulation in human ventral temporal cortex. Neuroimage 1052 218:116964. doi:10.1016/j.neuroimage.2020.116964 